\title{
Multi-Opponent Model of Weighing Used in Up-stream Equipment Weighing System
}

\author{
Rong-rong Zhang ${ }^{\mathrm{a}}$, Jin Chen ${ }^{\mathrm{b}}$, Mei-li Zhou ${ }^{\mathrm{c}}$, Yuan Li ${ }^{\mathrm{d}}$, Lu-jia Wang ${ }^{\mathrm{e}}$, and Hui \\ Wang $^{f}$ \\ College of Electronic and Communication Engineering, Tianjin Normal University, Tianjin, China \\ arongrongzhang023@163.com, bjwoods@163.com, 'c2432134038@qq.com, \\ d1152151948@qq.com, ${ }^{\mathrm{e}} 792723727 @ q q . c o m,{ }^{\mathrm{f}} 929280544 @ q q . c o m$,
}

\begin{abstract}
Keywords: Differential quantitative, up-stream equipment, impact component, aloft component.
\end{abstract}
\begin{abstract}
To overcome the defects of involute single spiral transmitter, which has a long time weighing and beyond the permissible error easily, we designed a double helix differential quantitative transmission system as the main structure of the up-stream equipment for automatic weighing and pushing of materials, the equipment used the spiral data acquisition-control device that the main core is $\Sigma$ - $\triangle \mathrm{ADC}$ (Sigma-Delta Analog-to-Digital Converter) and multi-component extraction model weighing algorithm, having a digital filter and characteristics analysis for the weight value, filter out the impact component from the weight information and add estimated aloft value to compensate aloft component. Shortening the time of weighing and increasing the weighing accuracy extremely through control precision grade of millisecond, prolonged failure-free operation of automatic weighing equipment, meanwhile, satisfied the requirements of all kinds of rubber tire production process.
\end{abstract}

\section{Introduction}

As more and more cars into people's lives, automotive and tire manufacturing industry also showed a rising trend. At present, the number of domestic tire manufacturers more than 600 , while the market demand for radial tires has exceeded 500 million $^{[1]}$, faced with such a demand expansion, competition, product quality of the rubber tire market, high-quality, high standards of production equipment for tire rubber manufacturer is essential. Up-stream equipment is a set of automated weighing ingredients, pneumatic conveying, mixing materials in one of the intelligent device, it is widely used electronic circuitry, mechanical design and automatic control technology ${ }^{[2]}$, the operating speed and control accuracy plays a vital role on the tire production.

The weighing of material with up-stream equipment on the rubber tire production line is using a combination of high precision screw conveyor and electronic scales for feeding and conveying. However, the existing the up-stream equipment load for a long time, limiting the freedom of the production process, but also easy to exceed the allowable error, resulting in the failure load. Therefore, it has an important practical value that developing the up-stream equipment of high precision based on $\Sigma-\triangle \mathrm{ADC}$, double helix conveyor for automatic weighing and pushing the material.

\section{2 overall production system architecture of up-stream}

The differential quantitative production systems in this paper, including the mechanical and hardware circuit, mainly by double screw conveyor and the up-stream equipment control system, which also includes a liquid crystal display, the interface and communications components.

\subsection{Architectural Overview of double helix conveyor}

The double helix conveyor is designed to double helix structure which includes the inlet, main spiral motor, main spiral, fuselage, vice spiral motor and vice spiral, the discharge port. Main spiral motor is placed under the bottom of the fuselage, and connected to the main spiral, main spiral mounted inside the fuselage horizontally, material is put into the main spiral from the inlet, and then 
pushed to vice spiral by the main spiral, vice spiral is connected to vice spiral motor through the pulley. The discharge port is disposed below the fuselage, and installed control valve for the material, the primary and secondary spiral share a spout. Which is designed to two main helical spiral, spiral using front blade, the pitch is $160 \mathrm{~mm}$, its pitch size suitable for the material to promote efficient, single-stage helical pitch, the pitch is $200 \mathrm{~mm}$, making the material flow evenly; Vice spiral diameter is about $80 \mathrm{~mm}-100 \mathrm{~mm}$, the coil cross-sectional area is $5027-7854 \mathrm{~mm}^{2}$. When the main spiral rotates, the material along the spiral ramp is pushed forward, and from the spout into the electronic scales, while vice spiral groove material is filled with material. Vice spiral rotates, push a small amount of material flowing out. The speed and on-off time of main spiral and vice spiral is controlled by the up-stream equipment system, do an efficient and accurate quantitative delivery.

\subsection{The up-stream equipment's components}

The up-stream equipment composed by the control unit, interactive, weighing sensor, digital In/Out module, Main spiral drive and vice spiral drive and so on. System architecture diagram is shown in Fig. 1.



Fig. 1 overall system architecture diagram

The system's main control unit constituted by high-precision 24-bit ADC with FPGA, FPGA is the logic control core of system, ADC is used to signal conversion. Human-computer interaction adopt advanced operation display panel based on $\mathrm{VC}++$ language, users can set the weight information, control accuracy requirements and other parameter values through interactive, and displays the resulting output. Weighing sensor located in the bottom of electronic scales to collect the weight information of materials on the electronic scale. Switching in/out module used to implement data collection and output control functions. Main spiral drive and vice spiral drive according to the instructions issued by the main control implementation planchip respectively control the main spiral motor and vice spiral motor's start-stop and operating speed.

When the operator set up related parameters in the HMI (Human Machine Interface), differential quantitative transmission system according to the operation command to start rotating double helix conveyor feeding, according to the target value to adjust spiral precessing speed, when the material is put into the electronic scale by the conveyor, the system will gather weight information from the load cell quickly, finally according to comprison result of the target value, the double helix precession state and the actual value of the acquisition, to control main spiral and vice spiral's coordinated campaign through the information output,and then, to carry out accurate quantitative differential feeding.

\section{Embodiments}

How to collect and process the weight informations, and precise control of spiral to complete the weighing and transport of materials is a key technology throughout the design.This design has proposed and established the information collect of spiral acquisition-control device based on embedded programs, implementation of high precision feeding and conveying of materials. Its overall structure is shown in Fig. 2. 


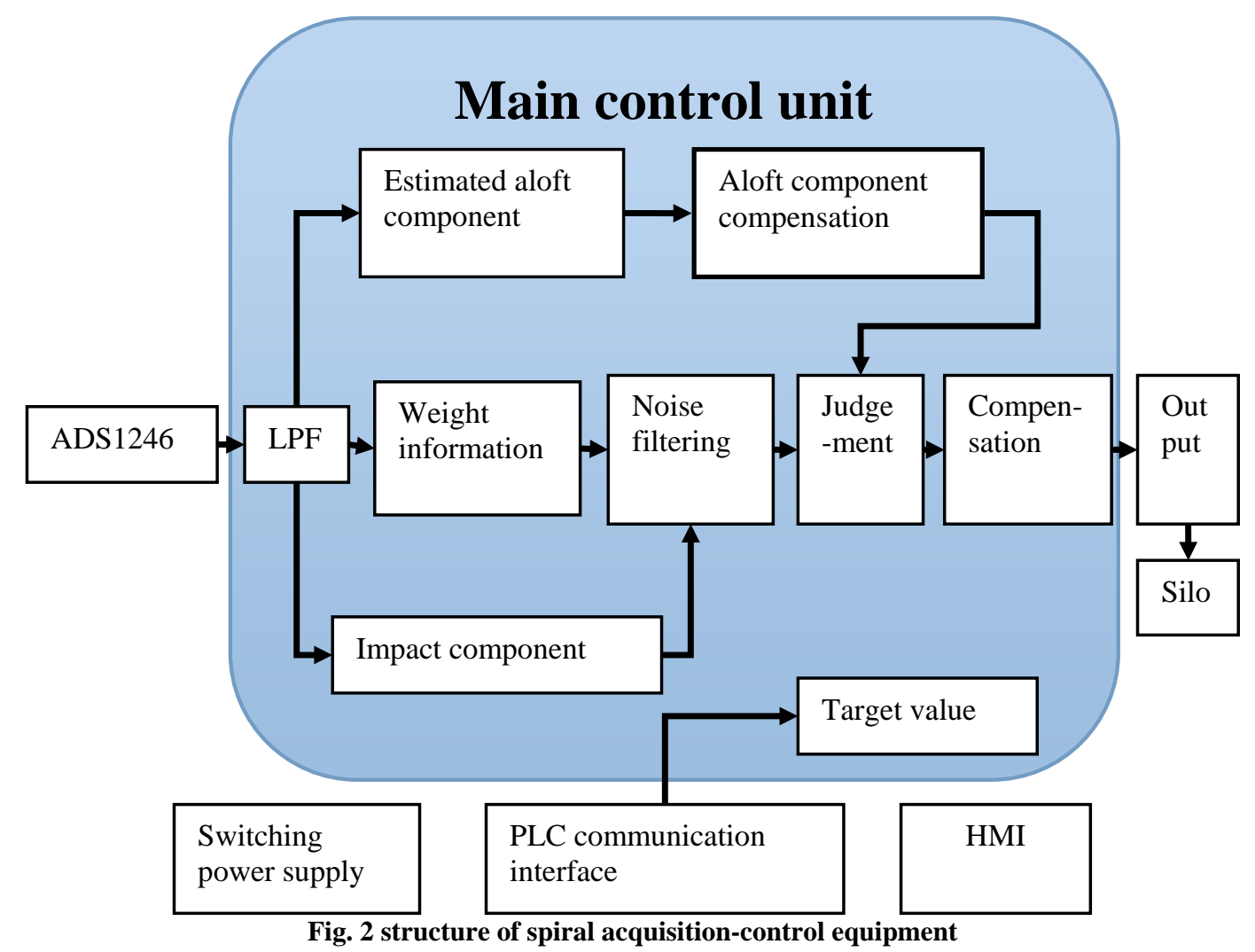

(1) Set up the target weight information in HMI, weighing control system started, the system will first judge the location of the main spiral and vice spiral information;

(2) The target weight values set by the user, as the target value of main control unit;

(3) The system start double-screw conveyors when it receives a start command, and recording start time value of the precession speed screw conveyors;

(4) When transmitting and weighing begin, ADS1246 quickly sample the processed sensor signal and digital quantification, then have the digital signal filtering and spectral analysis, establishing a multi-component models, namely weight information is extracted, the impact of information and air information;

(5) Weight information filtered Gaussian white noise and impulse noise and compensates for the average endurance components and validation;

(6) Analysis the location of the materials in spiral, endurance cycle variation is calculated, and variable compensation;

(7) According to the model, you can calculate the value of a more realistic weight, then this value is compared with the preset target value, than the result to control the starting and stopping of conveyor with rotating speed, eventually putting material into silo.

\section{Design of Multi-component Measurement Model}

In this design, the most outstanding technical content is establishing a multi-component measurement model which is related to weight information, impact information and aloft information. In the research process of design, we analysis and processing data, then extracting impact component and estimating aloft component, but also in consider of the Gaussion white noise that existing in the surrounding environment.

We construct a multi-component measurement model and has it verified. Eq.1 as follows:

$S(\mathrm{n})=a X_{1}(n)+b\left[C+\Delta f\left(X_{2}\right)\right]-c X_{3}(n)-\delta(n)$

$S(n)$ is the actual materials weight, $X_{1}(n)$ is collection value, $C+\Delta f\left(X_{2}\right)$ is the estimate aloft weight, aloft component is divided to average hang component $C$ and endurance cycle variable $\Delta f\left(X_{2}\right), X_{3}(n)$ is the impact component, $\delta(n)$ is the environmental noise component of the system. 
In the process of verify, at first, we have a low pass filter and graphical analysis for the output value of ADC, add estimate aloft component to weight value, and then filter out the impact component and various system noise. We use weight compensation constant theorem to calculate average hang component $C$, and spline function successive approximation obtain endurance cycle variable $\Delta f\left(X_{2}\right)$ that is a multi-stage function. As to $c X_{3}(n)$ and $\delta(n)$, we can filter out it through low-pass filter. In addition, as to three components coefficient of $a, b$ and $c$, will be preseted in system's storage media by technician on-site testing and calibrating.

\section{Control Signal Extraction}

Analog signal from the sensor is always accompanied by noise, and the presence of noise will increase measurement error, reduce the quality of the useful signal ${ }^{[3]}$, so we need to establish a weak signal processing system to access to the signal.

To weaken the system noise, we can use some similar technique to filter out noise from the signal, such as frequency-domain techniques, wavelet analysis, matched filtering and adaptive digital filtering, detection theory ${ }^{[4-5]}$. In this design, the basic idea to extract the signal is establishing an analog signal processing system, when the system collects the signal, it is subject to a basic level of amplification and low-pass filter, filtering the high frequency noise; secondly, oversampling and spectral shaping for signal by $\Sigma-\triangle \mathrm{ADC}$, pushing noise to high frequency with an integrator, which takes high frequency noise located outside the passband of the filter, and then having a secondary filter; finally, sending the processed signal into the FPGA, filtering the signal by FPGA that has a low-pass filter, get the ideal weak signal in the end. The filter is a multi-step linear phase finite impulse response low-pass filter.

\section{Conclusion}

This design uses a double helix conveyor and the double helix differential quantitative equipment to achieve precise weighing and transmission of materials. The signal through the multi-stage high-end precision digital filter to filter out the noise get better, improve signal to noise ratio of the entire control system. Meanwhile achieving complete FPGA combined with high-precision ADC as the secret training of auxiliary control unit, increases the accuracy of the entire control system chosen model from the design hardware and software aspects of the feasibility of its technology, the future machinery control is of great value significance.

\section{Acknowledgements}

Thanks for the supporting by Tianjin Edge Technology and Applied Basic Research Project (14JCYBJC15800), TJNUDP (52XK1206), TJNU - Student Entrepreneurial Innovation Program(201410065011) in China.

\section{References}

[1] Wang F, Wu GZ. Brief Introduction of Racial Tire Technology in China. TIRE INDUSTRY, 2006, 26(8):457-461. doi: 10.3969/j.issn.1006-8171.2006.08.002.

[2] Li Y. Development and Progress in Up-stream Equipment of Mixer. SPECIAL PURPOSE RUBBER PRODUCTS, 2003, 24(6):51-55.doi:10.3969/j.issn.1005-4030.2003.06.017.

[3] Tong SB, Hua CY. Analog Electronics Technical Foudation. Higher Education Press, 2006.

[4] Gao JJ. Weak Signal Detection. Beijing: Tsinghua University Press, 2011:1-39.

[5] Joseph Thomas Nabicht. High Frequency Continuous Time Filters for Mixed-signal Application[PhD Thesis]. Texas A\&M University, 2000. 